von Teilgebieten einer Fachrichtung (vgl. \$1 Nr. 3 Gesetz zur Änderung des BayKrG v. 23. 5.2006, Bay. GVB1. 295, m. W.v. 1.7.2006) selbst auf die Terminologie der WBO (vgl. Gesetzentwurf der Staatsregierung zur Änderung des BayKrG, Bay. LT-Dr. 15/3794 Teil B, S. 11 Zu Art 4).

3 . Auch die übrigen Voraussetzungen des Vergütungsanspruchs sind nach dem Gesamtzusammenhang der den erkennenden Senat bindenden unangegriffenen Feststellungen des LSG ( $\$ 163$ SGG) erfüllt. Die bei den Versicherten bestehenden Kniegelenkserkrankungen erforderten die stationär durchgeführten Knie-TEP-Operationen. Der K1. erfüllte auch die Anforderungen der Mindestmengenvereinbarung (MMV v. 16.8.2005 i.d.F. v. 11.11.2010, gültig ab 1.1.2011, BAnz. Nr. 181, S. 3976). Denn er überschritt in den Vorjahren 2009 und 2010 die Mindestmenge von 50 Knie-TEP-Operationen. Der Kl. berechnete für den stationären Aufenthalt rechtmäßig die DRG I44B die zu einem Vergütungsanspruch in Höhe von 7443,29 Euro und 7445,78 Euro führt. Bezüglich der Vergütungshöhe besteht zwischen den Beteiligten auch kein Streit.

4. Der Zinsanspruch ergibt sich aus $\$ 69$ Abs. 1 S. 3 SGB V i. V. mit $\int \$ 291,288$ Abs. 1 S. 2 BGB und $\$ 12$ Nr. 1 Abs. 1 und 2 der Vereinbarung für den Vereinbarungs-/ Pflegesatzzeitraum 2011 nach $\$ 11$ Abs. 1 KHEntgG und $\$ 17$ Abs. 1 BPflV. Danach beträgt die Zahlungsfrist für Krankenhausrechnungen drei Wochen. Ab Überschreitung der Zahlungsfrist sind Verzugszinsen in Höhe von vier Prozentpunkten zu zahlen. [...]

https://doi.org/10.1007/s00350-020-5472-8

\section{Anmerkung zu BSG, Urt. v. 9.4.2019 - B 1 KR 17/18 R (Bay. LSG)}

\section{Karin Hahne}

Die Entscheidung des BSG befasst sich materiell-rechtlich mit der Frage, ob die als orthopädisch einzustufende Behandlungsmaßnahme Knie-TEP dem chirurgischen Versorgungsauftrag eines Krankenhauses zugeordnet werden darf. Zum zweiten gibt das Urteil Auskunft zu der Frage, inwieweit das Landeskrankenhausplanungsrecht einer revisionsgerichtlichen Kontrolle unterliegt.

Es liegt in der Natur der Sache, dass das BSG selbst zu der materiell-rechtlichen Frage des Umfanges des Versorgungsauftrages nur in einem Absatz Stellung nimmt, während die verfahrensrechtlichen Fragen einen weitaus gröBeren Raum einnehmen, da das BSG feststellt, dass kein Raum für eine eigene Auslegung der Revisionsinstanz besteht, weil das LSG das Landesrecht selbst ausgelegt hat. Das BSG prüft die Auslegung des LSG unter drei Aspekten:

1. Verstoß gegen Bundesrecht

2. Verstoß gegen revisibeles Landesrecht

3. Verstoß gegen das grundgesetzliche Willkürverbot

$\mathrm{Zu}$ 1: Das BSG stellt fest, dass es nicht ersichtlich sei, dass das Bundesrecht überhaupt Vorgaben für die Krankenhausplanung in Bezug auf Fachgebiete macht. Eine solche bundesgesetzliche Regelung wäre mit Verfassungsrecht nicht vereinbar. Das nicht von der konkurrierenden Gesetzgebungszuständigkeit nach Art. 74 Abs. 1 Nr. 19a GG umfasste Krankenhausplanungsrecht fällt in die Gesetzgebungszuständigkeit der Länder (Art. 70 Abs. 1 GG). Demgemäß gebe es keine speziellen bundesrechtlichen Vorgaben für die Länder, beim Krankenhausplanungsrecht auf die Fachgebiete der Weiterbildungsordnung Bezug zu nehmen.

Rechtsanwältin Dr. iur. Karin Hahne,

Fachanwältin für Medizinrecht, HFBP Rechtsanwälte und Notar, Friedrich-Ebert-Anlage 18, 60325 Frankfurt am Main, Deutschland
Zu 2: Nach $₫ 162$ SGG unterliegt Landesrecht nur dann einer revisionsgerichtlichen Kontrolle, wenn sich sein Geltungsbereich über den Bezirk des Berufungsgerichtes hinaus erstreckt. Daran fehlt es nach den Feststellungen des BSG für das bay. Landeskrankenhausplanungsrecht. Dass der bay. Landeskrankenhausplan nach der Auslegung durch das LSG auf die Gebietsbezeichnung der WBO Bezug nimmt, die in anderem Zusammenhang einer revisionsgerichtlichen Kontrolle unterliegt, begründet nicht die Revisibilität der landesrechtlichen Vorschriften. Es sei ohne Belang, dass die WBO aufgrund ihrer Orientierung an der Musterweiterbildungsordnung in Fragen der Fachfremdheit als inhaltsgleiche Regelung mit anderem Landesrecht einer revisionsgerichtlichen Kontrolle zugänglich wäre.

$\mathrm{Zu}$ 3: Erst bei der Prüfung, ob nicht insofern ein Ausnahmefall vorläge, in dem das Revisionsgericht trotz Irreversibilität der landesrechtlichen Vorschriften an die Auslegung des LSG nicht gebunden sei, weil die Auslegung des Willkürgebotes das Grundgesetz verletzt, kommt das BSG $\mathrm{zu}$ einer eigenen Feststellung zu der materiell-rechtlichen Frage. Nach Auffassung des BSG sei das Willkürverbot erst verletzt, wenn sich das Berufungsgericht soweit von den gesetzlichen Vorgaben entfernt hat, dass sich ein vernünftiger, sich aus der Natur der Sache ergebender oder sonst wie sachlich einleuchtender Grund für die gewählte Auslegung nicht finden lässt. Dies sei vorliegend nicht der Fall. Das LSG stützt sich bei dem offenen Wortlaut des bay. Krankenhausbedarfsplans und des Feststellungsbescheides hinsichtlich der Fachrichtung auf ungeschriebene Grundsätze, die später ausdrücklich in die Regelungen übernommen worden sind, nämlich den Verweis auf die Fachgebietsgrenzen in der Weiterbildungsordnung. Das BSG stellt ausdrücklich fest: „Soweit das Krankenhausplanungsrecht eines Landes auf Gebietsbezeichnungen vergleichbar jenen der Weiterbildungsordnung abstellt, liegt ein Sachzusammenhang nicht fern". Abschließend stellt das BSG fest, dass auch die übrigen Voraussetzungen des Vergütungsanspruches, wie insbesondere die Anforderung an die Mindestmengenvereinbarung eingehalten worden seien.

Fazit: Trotz der nur marginalen eigenen Ausführungen zu der Frage, ob ein als orthopädisch einzustufender Eingriff, eine Knie-TEP, dem chirurgischen Versorgungsauftrag eines Krankenhauses zuzuordnen ist, darf nach diesem Urteil doch festgehalten werden, dass das BSG die Orthopädie und Unfallchirurgie als Unterfall eines chirurgischen Versorgungsauftrages bewertet. Im vertragsärztlichen $\mathrm{Be}-$ reich ist dies in der Bedarfsplanung mit Beschluss des G-BA v. 20.9.2018 inzwischen auch nachvollzogen worden.

\section{Zugang zur manuellen Therapie nur für Physiotherapeuten eröffnet}

SGB V §§91, 92, 124, 125, 138

Das auf ausgebildete Physiotherapeuten beschränkte Weiterbildungserfordernis für Leistungen der manuellen Therapie, das Masseure/medizinische Bademeister von der Erbringung dieser Leistungen zu Lasten der gesetzlichen Krankenversicherung grundsätzlich ausschließt, enthält für diese Berufsgruppe eine gerechtfertigte und zumutbare Einschränkung ihrer Berufsausübungsfreiheit.

BSG, Urt. v. 16.3.2017, B 3 KR 24/15 R (LSG Bad.-Württ.) 